\title{
Heterogeneous Ontology Merging Using Formal Concept Analysis
}

\author{
Jaturada Deeying $^{+}$and Wiwat Vatanawood \\ Department of Computer Engineering \\ Faculty of Engineering, Chulalongkorn University, Bangkok, Thailand
}

\begin{abstract}
The increasing of ontology usage caused some overlapping ontologies which motivates knowledge engineers to enable the collaborative use of similar ontologies. It introduces the concept of ontology merging. Unfortunately, there are a few researches on heterogeneous ontology merging algorithm and most of them operated in the same or similar domain ontologies. In this paper, we aimed to merge heterogeneous ontologies on relevant domain using Formal Concept Analysis. We took DrugBank and Disease ontologies as our case study. The relationships between drug and disease are presented in this paper in the form of formal lattice. The result of this paper is the merged ontology of the given use case. The result was evaluated by comparing with the medication suggestion. The evaluation shows that the proposed method yielded a correct suggestion accordingly and it will be useful to support knowledge engineer to merge ontologies using corresponded attributes between ontologies.
\end{abstract}

Keywords: formal concept analysis, ontology merging, heterogeneous ontology merging

\section{Introduction}

Ontologies have been established to construct the conceptual model for the domain of interest [1]. These used to share the understanding of knowledge and its relationship among each other. Many researchers in various area, e.g. medicine, agriculture and environmental science tried to formulate and answer some ontology-related research questions, for instance, they tried to find the influence of environmental factors on human health and diseases in drug discovery research [2]. As of the growing usage of ontologies, the overlapping of existing ontologies occurs. Researchers take advantages of existing ontologies for a new purpose by reusing and integrating them together. But the integration of ontologies considers a challenging process because of the different formats, languages, and structures. Consequently, the interoperability of ontologies is obstructed by its nature characteristic - the heterogeneity.

Previous research works have applied Formal Concept Analysis (FCA) to support the knowledge engineer in analyzing and extracting conceptual hierarchy from a given domain of interest. Furthermore, FCA can also be used for merging independently ontologies [3]. However, those researches have focused on the same domain, and tried to improve only in the Concept Similarity Matching technique.

In this paper, we aimed to propose an ontology merging method for heterogeneous ontologies. We adopted an FCA approach and apply it on relevant domain knowledge using DrugBank and Disease ontology as our case study. We demonstrate the proposed method with an example of a viral and bacterial infectious disease, that supportive symptom treatment. We considering disease symptom matching with correspondence drug indication.

The following sections in this paper organized as follows: In section 2, we leverage a background of the ontology concept, ontology matching, ontology merging as long as the concept of FCA. After that, we review the literature on ontologies merging using FCA in section 3. The section 4 describes our proposed method. Lastly, the section 5 concludes our result and states the future work.

\footnotetext{
+ Corresponding author.

E-mail address: Jaturada.d@ student.chula.ac.th.
} 


\section{Background}

\subsection{Ontology}

In the context of computer and information sciences, ontology is an explicit description of the domain of interest [4]. The major components of an ontology consist of concepts (or classes), attributes (or properties), relations, and instance. Concept represents a set of things in the domain of interest, attribute describes characteristic of concept, relation describes the interactions between concepts or concept's properties, and instance is the concrete entities hold the inherited attributes of its related concept [5][6]. Ontology is a semantic way to share the structure of information. Recently, ontologies have been adopted in many business and scientific communities [7]. The ontology integration is the state-of-the-art way to share or reuse ontologies which applied the process of ontology matching and ontology merging. The definition and the detail process are described as follows.

- Ontology Matching is a process of which two (or more) ontologies are fed in and interpreted for a set of correspondences between a pair of similar concepts semantically. These matching are also called ontology mappings [8].

- Ontology Merging is a process of finding and merging two (or more) concepts that consider as a semantic similar into a single concept in order to create a new ontology from two ontology initiatives.
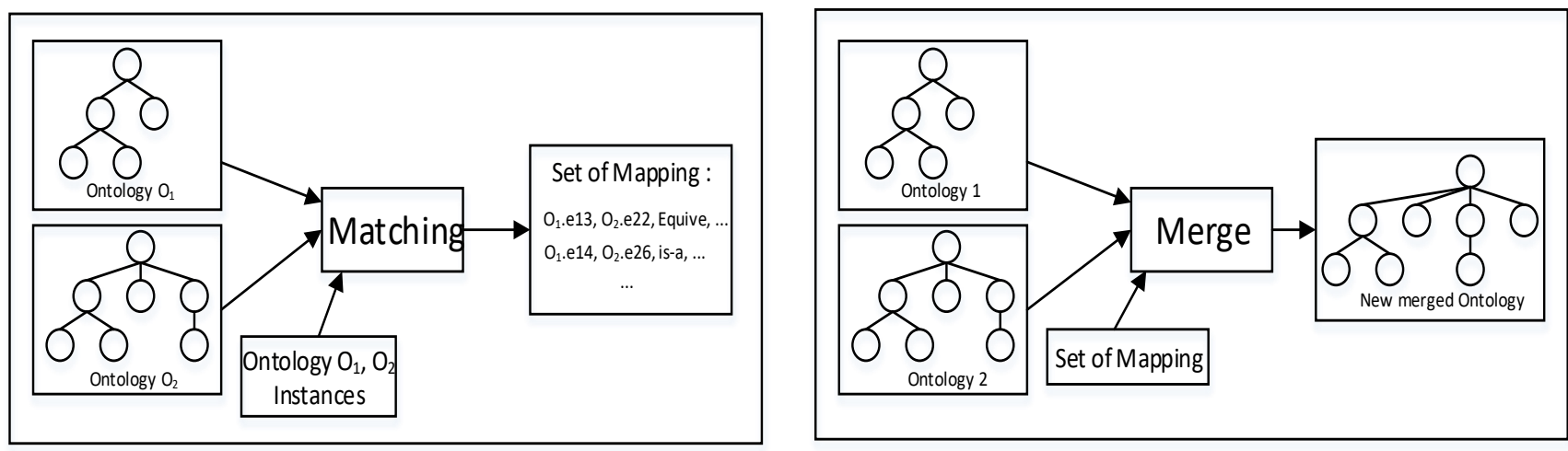

Fig. 1: A comparison between ontology matching (left) and ontology merging (right)[9]

\subsection{Formal concept analysis}

FCA was introduced in 1982 by Rudolf Wille [10]. The theoretical foundation of FCA was built on the Applied Lattice Theory and Set Theory [10] which deals with the concepts and concept hierarchies. FCA method takes the ontology data (in a matrix format - called 'formal context') and analyzes the association between concepts as a set of objects and a set of shared attributes [11]. The FCA yields two sets of sustainable outcomes. 1) A set of hierarchical relationships of concepts which called a Concept Lattice. 2) A set of interdependencies between attributes in the formal context.

- Formal Context

Definition 1: A formal context defined as a triple $K:=(O, A, R)$ where $O$ is a set of objects, $A$ is a set of attributes (properties), and $R$ is a binary association between $O$ and $A$ (i.e. $R \subseteq O \times A$ ) denoted by $(o, a) \in R$ or $(o, a) R$. Those notions can be referred as "object $o$ has attribute $a$ " or "the attribute $a$ applies to the object $o$ ".

- Formal Concept

Definition 2: For all formal context $(O, A, R)$, the definition of concept-forming operator $E \subseteq O$ and $I \subseteq A$ as in Eq. 1 and Eq. 2 respectively,

$$
\begin{aligned}
& E^{\prime}:=\{a \in A \mid \text { for each } o \in E:(o, a) \in R\} \\
& \mathrm{I}^{\prime}:=\{o \in O \mid \text { for each } a \in I:(o, a) \in R\}
\end{aligned}
$$

$E^{\prime}$ represents a set of all attributes shared by all objects from set $E$, and $I^{\prime}$ represents a set of all objects which share all the attributes from set $I$. A formal concept of any formal context $(O, A, R)$ is a pair $(E, I)$ where $E \subseteq O, I \subseteq A$, for which $E^{\prime}=I$ and $I^{\prime}=E$. The $E$ and $I$ sets are called the extent and the intent respectively. 
- Concept Lattice

Definition 3: A Concept Lattice is a structure which defined as a tuple of $(B(O, A, R), \leq)$, where $B(O, A, R)$ is a collection of all formal concepts of a given context and $\leq$ is a partial order relation between concepts.

A partial order relation for formal concepts $(A 1, B 1)$ and $(A 2, B 2)$ in a formal context $(O, A, R)$ holds that $(A 1, B 1) \leq(A 2, B 2)$ if and only if $A 1 \subseteq A 2$ and $B 2 \subseteq B 1$. It also describes the Subconcept/Superconcept relationship. All the formal concepts and relations can be transformed to a concept lattice structure which presented as a Hesse (line) diagram. Table 1 shows the formal context represented in matrix describing a set of objects (drug name) against a set of attributes (drug indication) and indicating the association between objects and attributes by " $\mathrm{X}$ " mark.

Table 1: An example of drug formal context

\begin{tabular}{|l|c|c|c|c|c|c|}
\hline \multirow{2}{*}{ Drug Name } & \multicolumn{7}{c|}{ Drug indication } \\
\cline { 2 - 7 } & Diarrhea & Fever & Headache & Vomiting & Nausea & Coughing \\
\hline Acetaminophen & & $\mathrm{x}$ & & & & \\
\hline Choline salicylate & & $\mathrm{x}$ & $\mathrm{x}$ & & & $\mathrm{x}$ \\
\hline Dextromethorphan & & & & & & $\mathrm{x}$ \\
\hline Dimenhydrinate & & & & $\mathrm{x}$ & $\mathrm{x}$ & \\
\hline Doxylamine & & & & $\mathrm{x}$ & & \\
\hline Ibuprofen & & $\mathrm{x}$ & $\mathrm{x}$ & & & \\
\hline Norfloxacin & $\mathrm{x}$ & & & & & \\
\hline
\end{tabular}

To depict the associations and relationships between objects and attributes, A concept lattice, as in Fig. 2, is the diagrammatic representation of the formal context in Table 1.

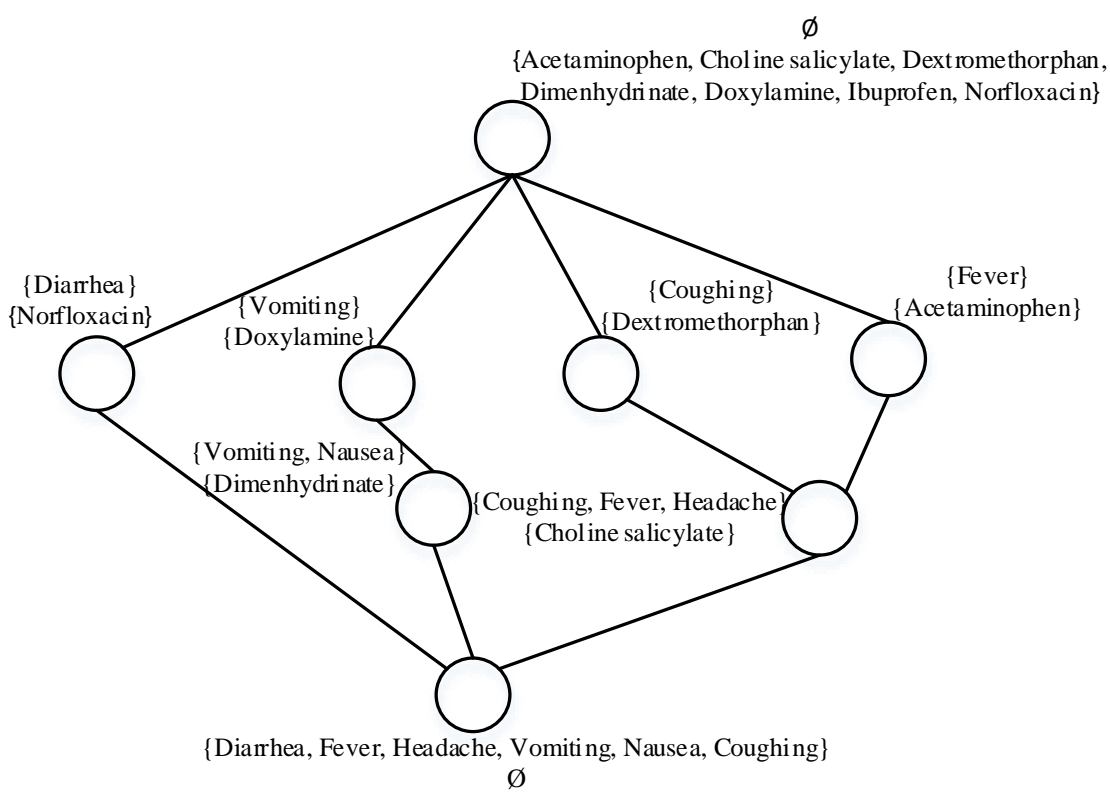

Fig. 2: An example of drug concept lattice

\section{Related Work}

In this section, we reviewed a broad list of literature in the area of ontology merging using FCA in order to state the current status of our research. FCA-MERGE for merging ontologies with a bottom-up approach was proposed by [12]. They applied techniques from natural language processing and formal concept analysis. Their merging process starts with extracting instances from source ontologies and a set of documents based on natural language processing. It yields two formal contexts from the extraction. Then, FCA-MERGE algorithm merges those formal contexts using FCA and computes for concept lattice. Lastly, their research reported merged ontologies from concept lattice with human interaction. Their approach tackled the problem of less semantic in current systems, but their method lacks flexibility. It required to collect domain documents that are relevant to source ontologies for extracting instances in the first step.

A new method for merging ontology based on formal concept analysis and WordNet - called FCAOntMerge was proposed by [13]. Their method merges the ontologies in four steps, which are: 1) Formulate 
the source ontologies in OWL format. 2) Extract concepts and attributes of ontologies and determine the formal context. 3) Match the attributes in the formal context using the antonym, synonym, hypernym, and hyponym in WordNet. 4) Associate formal contexts using Concept Lattice Merging algorithm and generate resulted concept lattice. Their research tried to solve the problem in only a specific domain, but it uses different format of ontology merging. Their research also enhanced merging efficiency using a discernibility matrix algorithm.

An ontology merging approach based on concept similarity was proposed by [14]. They were using FCA technique with WordNet. Their merging algorithm consists of five essential steps, which are: 1) Analyze source ontologies and formulate the formal context of each ontology. 2) Determine semantic similarity between concepts in formal context using WordNet and compute similarity score with its distance, density and depth as the factors of calculation. 3) Reduce redundancy of concepts and attributes in formal context. 4) Construct the concept lattice from reduced formal context. 5) Construct merged ontology from concept lattice. Their research is one of the works that applies concept similarity calculation on the concept merging algorithm.

From these researches, it reveals that there are several algorithms for merging concepts of ontology based on FCA. However, these researches have scoped on the same or similar domain. On the other hand, we found out that the concept similarity yields a measurable quantitative score which is objective to the context. Therefore, we decided to apply their method to merge heterogeneous ontology in relevant context using DrugBank and Disease ontology as our case study.

\section{Proposed Method}

With the result of method selection in literature review section, this section shall illustrate and describe how we tried to merge heterogeneous ontologies based on FCA using DrugBank and Disease ontology as our case study. To give an overview of the method, an activity diagram, as in Fig. 3, shows five major steps of our proposed merging method.

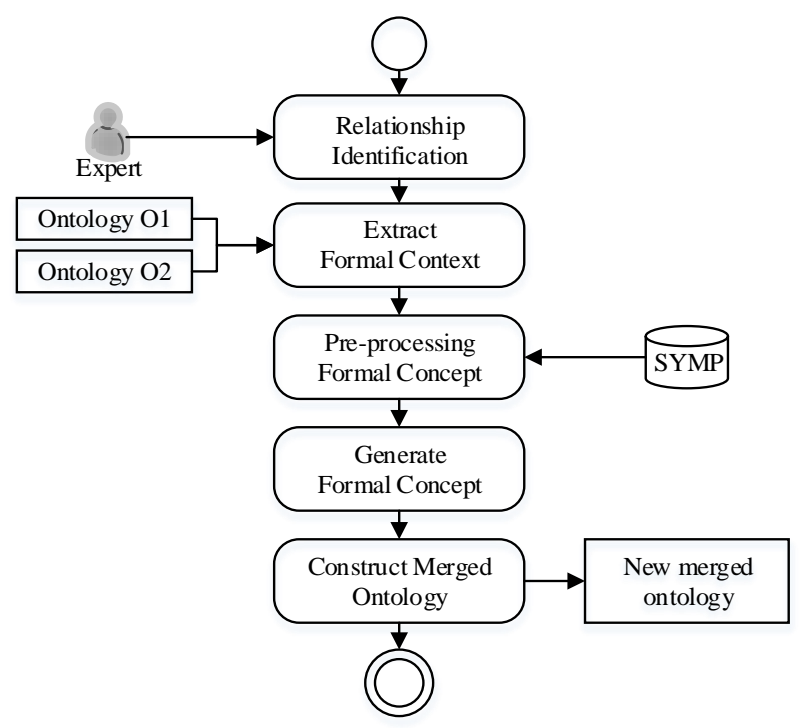

Fig. 3: An overview of the research methodology

\subsection{Relationship identification}

As our first step, we carefully gathered and assured that the input data is precise and correct. We asked the pharmaceutical and medical experts to identify relevant concepts required for the merge and define the name of relations among them. Then, we identified corresponding attributes for matching between DrugBank and Disease ontologies. For instance, "may be treated by" and "may treat" relations are identified to establish new directional relationships between diseases and relevant drugs. We also identified "drug structured indication" attribute to consider the conformance with "disease symptom". The result of this step shows in Fig. 4. 


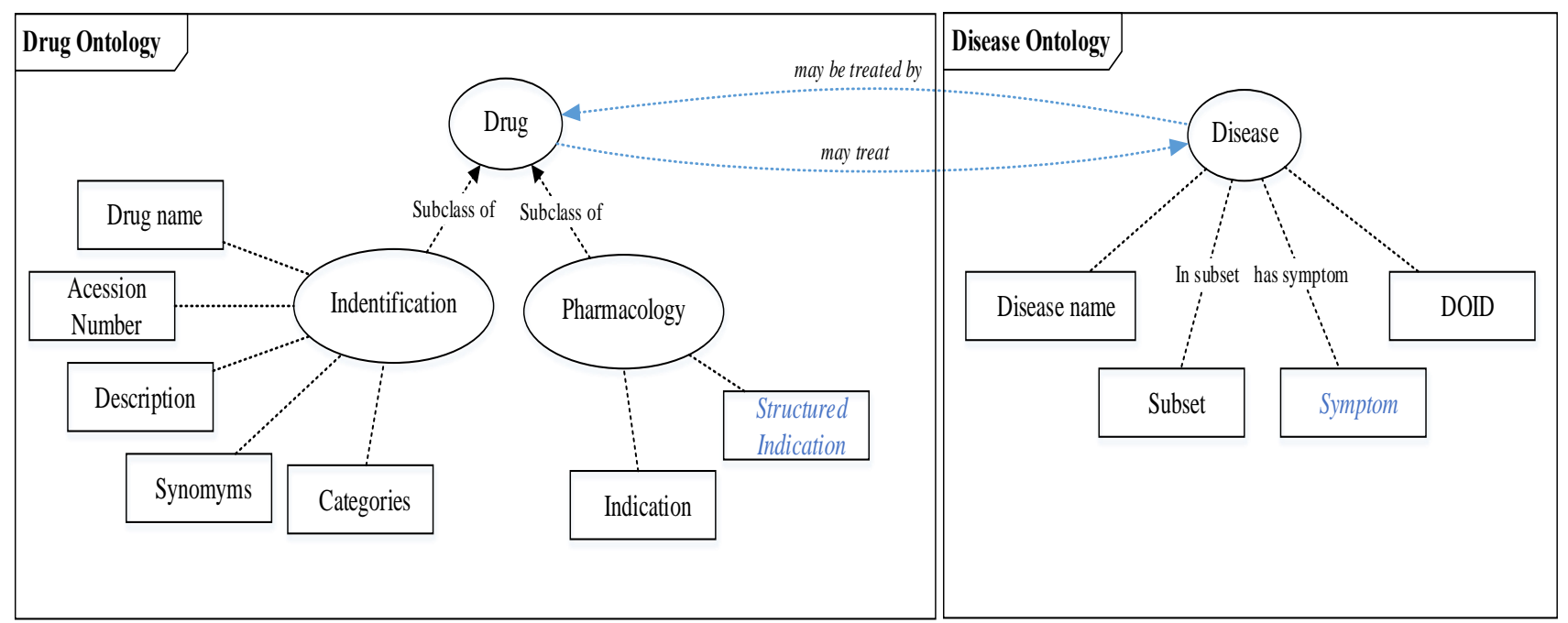

Fig. 4: The identified relationships among drug and disease ontologies

\subsection{Formal context extraction}

From the identified relationships of drug and disease from the previous step, we tried to produce a formal context of the relationship by following the steps defined as shown in Fig. 5. Initiatively, we constructed a context table from those relationships by extracting classes, attributes, and instances. The resulted context tables for drug and disease showed in Table 2 and Table 3 respectively. Subsequently, we integrated these tables by matching symptoms together as result in Table 4 .

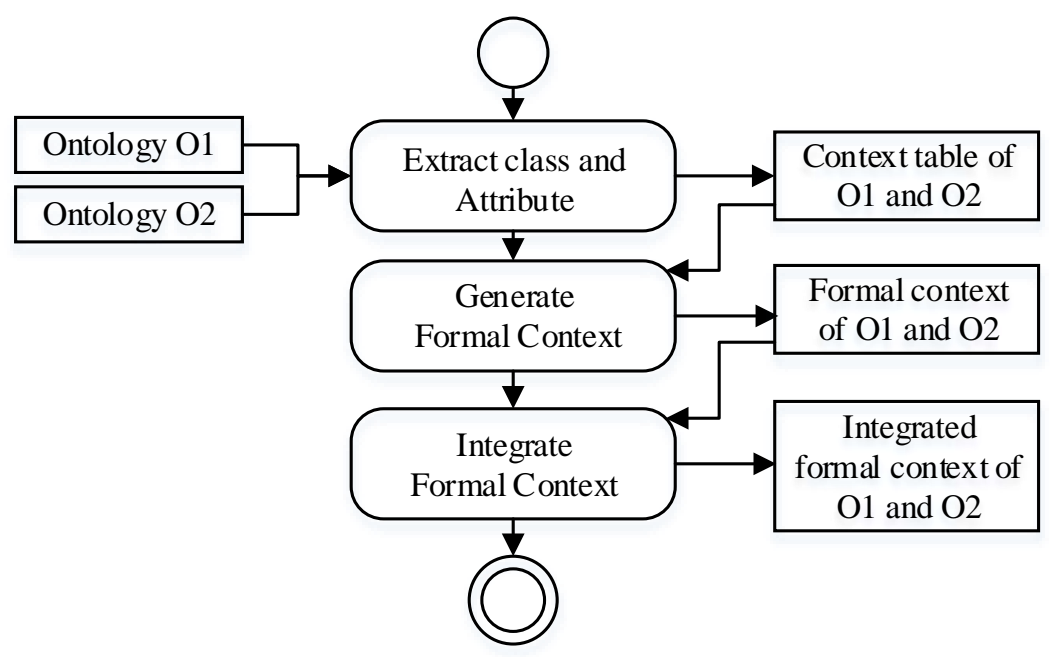

Fig. 5: An overview of formal context extraction procedure

Table 2: An example of disease context table

\begin{tabular}{|l|l|l|}
\hline \multicolumn{1}{|c|}{ Disease ID } & \multicolumn{1}{|c|}{ Disease } & \multicolumn{1}{c|}{ Symptom } \\
\hline DOID:10841 & Eastern equine encephalitis & Seizures \\
\cline { 3 - 3 } & & Vomiting \\
\hline \multirow{2}{*}{ DOID: 11103} & Rickettsialpox & Fever \\
\cline { 3 - 3 } & & Headache \\
\cline { 3 - 3 } & & Myagia \\
\hline DOID:11573 & Listeriosis & Diarrhea \\
\cline { 3 - 3 } & & Fever \\
& & Headache \\
\cline { 3 - 3 } & & Vomiting \\
\hline
\end{tabular}


Table 3: An example of drug context table

\begin{tabular}{|l|l|l|}
\hline $\begin{array}{c}\text { Drug Bank } \\
\text { ID }\end{array}$ & \multicolumn{1}{|c|}{ Drug } & \multicolumn{1}{|c|}{$\begin{array}{c}\text { Structured } \\
\text { Indication }\end{array}$} \\
\hline DB00181 & Baclofen & Muscle pains \\
\cline { 3 - 3 } & & Arthragia \\
\hline DB00366 & Doxylamine & Vomiting \\
\hline DB01050 & Ibuprofen & Fever \\
\cline { 3 - 3 } & & Headache \\
\cline { 3 - 3 } & & Migraine \\
\hline DB01059 & Norfloxacin & diarrhea \\
\hline DB00252 & Phenytoin & Seizures \\
\hline
\end{tabular}

Table 4: A result table of formal context integration

\begin{tabular}{|c|c|c|c|c|c|c|c|c|c|c|}
\hline \multirow[t]{2}{*}{ concept } & \multirow[t]{2}{*}{ Instance } & \multicolumn{9}{|c|}{ Symptom / Structured Indication } \\
\hline & & Arthragia & Diarrhea & Fever & Headache & Migraine & Myagia & $\begin{array}{c}\text { Muscle } \\
\text { Pain }\end{array}$ & Seizures & Vomiting \\
\hline \multirow[t]{3}{*}{ Disease } & DOID:10841 & & & & & & & & $\mathrm{x}$ & $\mathrm{x}$ \\
\hline & DOID:11103 & & & $\mathrm{x}$ & $\mathrm{x}$ & & $\mathrm{x}$ & & & \\
\hline & DOID:11573 & & $\mathrm{x}$ & $\mathrm{X}$ & $\mathrm{x}$ & & & & & $\mathrm{x}$ \\
\hline \multirow[t]{11}{*}{ Drug } & DB00316 & & & $\overline{\mathrm{X}}$ & & & & & & \\
\hline & DB00181 & $\mathrm{x}$ & & & & & & $\mathrm{x}$ & & \\
\hline & DB01744 & & & & & & & $\mathrm{x}$ & & \\
\hline & DB00537 & & $\mathrm{x}$ & & & & & & & \\
\hline & DB00366 & & & & & & & & & $\mathrm{x}$ \\
\hline & DB01050 & & & $\mathrm{x}$ & $\mathrm{x}$ & $\mathrm{x}$ & & & & \\
\hline & DB00788 & & & $\mathrm{x}$ & $\mathrm{x}$ & & & & & \\
\hline & DB01059 & & $\mathrm{x}$ & & & & & & & \\
\hline & DB00252 & & & & & & & & $\mathrm{x}$ & \\
\hline & DB13747 & & & & & & & $\mathrm{x}$ & & \\
\hline & DB00313 & & & & & & & & $\mathrm{x}$ & \\
\hline
\end{tabular}

\subsection{Formal concept pre-processing}

Actually, the result from the previous step is enough for construct the formal concept based on the formal context. However, it may possible that some attributes from different ontologies are redundant. For example, in this case study, some symptoms are semantically similar. To enhance the correctness and precision of the result, the formal context should be pre-processed by reducing the redundancy before continuing to the next step. The overview of the pre-processing activities is described in the following activity diagram.

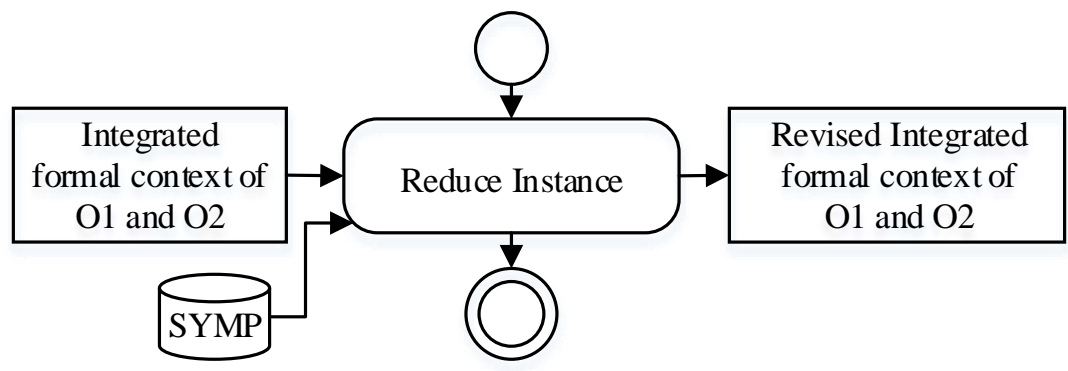

Fig. 6: An overview of the formal concept pre-processing

For our case study, we used SYMP vocabulary which is a standard vocabulary for disease symptom as our reference. The pre-processing reveals that there are redundancies of symptom attributes which are the same semantically. For instance, the "Myagia" symptom from disease context is the synonym of "Muscle Pain" from drug context. Thus, we considered the redundant attributes as a single attribute and we revised the formal context table as shown in Table 5. 
Table 5: A result table of formal context without redundancy

\begin{tabular}{|c|c|c|c|c|c|c|c|c|c|}
\hline \multirow[t]{2}{*}{ concept } & \multirow[t]{2}{*}{ Instance } & \multicolumn{8}{|c|}{ Symptom / Structured Indication } \\
\hline & & Arthragia & Diarrhea & Fever & Headache & Migraine & Muscle Pain & Seizures & Vomiting \\
\hline \multirow[t]{3}{*}{ Disease } & DOID: 10841 & & & & & & & $\mathrm{X}$ & $\mathrm{x}$ \\
\hline & DOID:11103 & & & $\mathrm{X}$ & $\mathrm{X}$ & & $\mathrm{X}$ & & \\
\hline & DOID:11573 & & $\mathrm{x}$ & $\mathrm{X}$ & $\mathrm{X}$ & & & & $\mathrm{x}$ \\
\hline \multirow[t]{11}{*}{ Drug } & DB00316 & & & $\mathrm{X}$ & & & & & \\
\hline & DB00181 & $\mathrm{X}$ & & & & & $\mathrm{x}$ & & \\
\hline & DB01744 & & & & & & $\mathrm{x}$ & & \\
\hline & DB00537 & & $\mathrm{x}$ & & & & & & \\
\hline & DB00366 & & & & & & & & $\mathrm{x}$ \\
\hline & DB01050 & & & $\mathrm{X}$ & $\mathrm{X}$ & $\mathrm{X}$ & & & \\
\hline & DB00788 & & & $\mathrm{x}$ & $\mathrm{x}$ & & & & \\
\hline & DB01059 & & $\mathrm{x}$ & & & & & & \\
\hline & DB00252 & & & & & & & $\mathrm{x}$ & \\
\hline & DB13747 & & & & & & $\mathrm{X}$ & & \\
\hline & DB00313 & & & & & & & $\mathrm{X}$ & \\
\hline
\end{tabular}

\subsection{Formal concept construction}

After the pre-processing, the formal context is ready for constructing the formal concept. We started with simplifying the wording and texts in the formal context for ease of representation. We used D, Dr, and $\mathrm{S}$ to abbreviate Disease, Drug, and Symptom respectively. The simplification results in Table 6. Then, we formulated the interdependent relationships between concepts and attributes in the form of $(o, a)$ tuple. To understand how the formulation result produce, we match the Dr9 and Dr11 as an example. From Table 6, it shows that both Dr9 and Dr11 contained S7 attribute and the resulted tuple is ( $\{\mathrm{Dr} 9, \operatorname{Dr} 11\},\{\mathrm{S} 7\})$. The result of the formal concept construction is shown in the Table 7.

Table 6: A simplified formal context table

\begin{tabular}{|c|c|c|c|c|c|c|c|c|c|}
\hline \multirow[t]{2}{*}{ concept } & \multirow[t]{2}{*}{ Instance } & \multicolumn{8}{|c|}{ Symptom / Structured Indication } \\
\hline & & S1 & $\mathrm{S} 2$ & S3 & $\mathrm{S} 4$ & S5 & S6 & S7 & S8 \\
\hline \multirow[t]{3}{*}{ Disease } & D1 & & & & & & & $\mathrm{x}$ & $\mathrm{x}$ \\
\hline & D2 & & & $\mathrm{x}$ & $\mathrm{X}$ & & $\mathrm{x}$ & & \\
\hline & D3 & & $\mathrm{x}$ & $\mathrm{x}$ & $\mathrm{x}$ & & & & $\mathrm{x}$ \\
\hline \multirow[t]{11}{*}{ Drug } & Dr1 & & & $\overline{\mathrm{x}}$ & & & & & \\
\hline & Dr2 & $\mathrm{x}$ & & & & & $\mathrm{x}$ & & \\
\hline & Dr3 & & & & & & $\mathrm{x}$ & & \\
\hline & Dr4 & & $\mathrm{x}$ & & & & & & \\
\hline & Dr5 & & & & & & & & $\mathrm{x}$ \\
\hline & Dr6 & & & $\mathrm{x}$ & $\mathrm{x}$ & $\mathrm{x}$ & & & \\
\hline & Dr7 & & & $\mathrm{x}$ & $\mathrm{x}$ & & & & \\
\hline & Dr8 & & $\mathrm{x}$ & & & & & & \\
\hline & Dr9 & & & & & & & $\mathrm{x}$ & \\
\hline & Dr10 & & & & & & $\mathrm{x}$ & & \\
\hline & Dr11 & & & & & & & $\mathrm{X}$ & \\
\hline
\end{tabular}

Table 7: A formal concept of drug and disease ontologies merging

\begin{tabular}{|l|l|}
\hline No. & \multicolumn{1}{|c|}{ Formal concepts } \\
\hline 1 & $(\emptyset,\{\mathrm{S} 1, \mathrm{~S} 2, \mathrm{~S} 3, \mathrm{~S} 4, \mathrm{~S} 5, \mathrm{~S} 6, \mathrm{~S} 7, \mathrm{~S} 8\})$ \\
\hline 2 & $(\{\mathrm{D} 3\},\{\mathrm{S} 2, \mathrm{~S} 3, \mathrm{~S} 4, \mathrm{~S} 8\})$ \\
\hline 3 & $(\{\mathrm{Dr} 6\},\{\mathrm{S} 3, \mathrm{~S} 4, \mathrm{~S} 5\})$ \\
\hline 4 & $(\{\mathrm{D} 2\},\{\mathrm{S} 3, \mathrm{~S} 4, \mathrm{~S} 6\})$ \\
\hline 5 & $(\{\mathrm{Dr} 7\},\{\mathrm{S} 3, \mathrm{~S} 4\})$ \\
\hline 6 & $(\{\mathrm{D} 1\},\{\mathrm{S} 7, \mathrm{~S} 8\})$ \\
\hline 7 & $(\{\mathrm{Dr} 2\},\{\mathrm{S} 1, \mathrm{~S} 6\})$ \\
\hline 8 & $(\{\mathrm{D} r\}\},\{\mathrm{S} 8\})$ \\
\hline
\end{tabular}




\begin{tabular}{|l|l|}
\hline 9 & $(\{$ Dr9,Dr11 $\},\{$ S 7$\})$ \\
\hline 10 & $(\{$ Dr4,Dr8 $\},\{$ S2 $\})$ \\
\hline 11 & $(\{$ Dr3,Dr10,$\{$ S6 $\})$ \\
\hline 12 & $(\{$ Dr1 $\},\{$ S3 $\})$ \\
\hline 13 & $(\{$ D1,D2,D3,Dr1,Dr2,Dr3,Dr4,Dr5,Dr6,Dr7,Dr8,Dr9,Dr10,Dr11 $\}, \varnothing)$ \\
\hline
\end{tabular}

\subsection{Concept lattice formulation and merged ontology construction}

In the last step, we generated a Concept Lattice from formal concept and relationship tuple. The concept lattice, in this case, is represented in the diagram as shown in Fig. 7. The node represents the pair of relationships among concepts and attributes, and the edge represents the similar relationship of two nodes. For example, the node ( $\{\mathrm{Dr} 7\},\{\mathrm{S} 3, \mathrm{~S} 4\})$ has an edge to $(\{\mathrm{D} 3\},\{\mathrm{S} 2, \mathrm{~S} 3, \mathrm{~S} 4, \mathrm{~S} 8\})$ since it possesses the same attributes, i.e. S3 and S4.

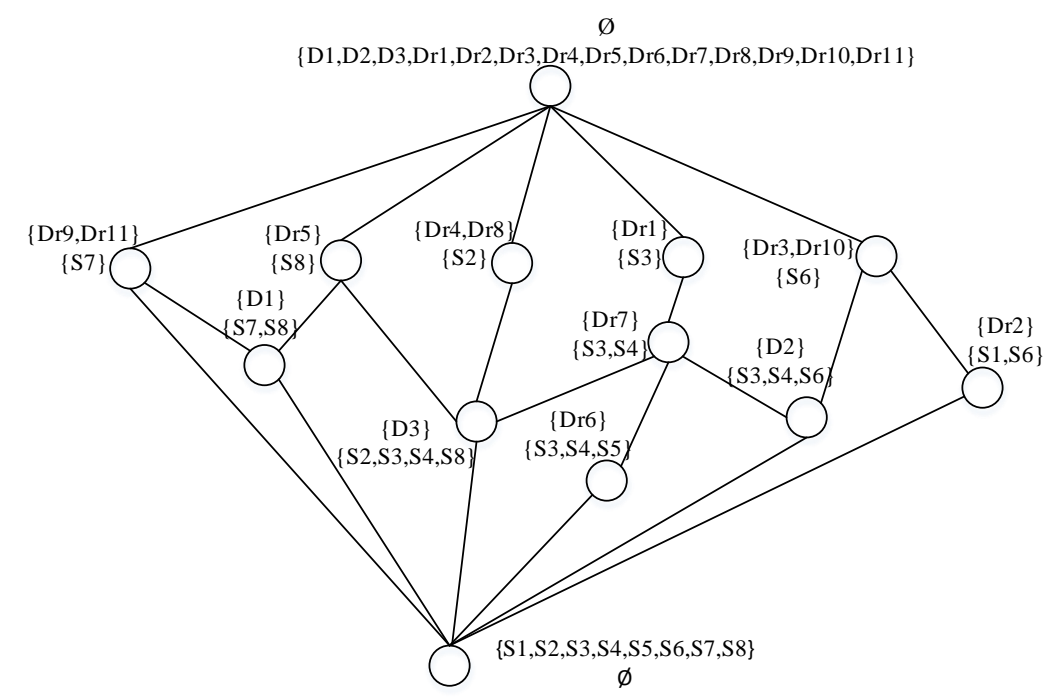

Fig. 7: A resulted concept lattice of merged drug and disease ontologies

At the end of the day, the concept lattice can be used in constructing the new merged ontology. To clarify the example of the merged ontology for our use case, we selected "Phenytoin" drug (Dr9) as an example. The concept lattice can support in constructing the ontology for the "Phenytoin (Dr9)" drug treat a "Seizure Symptom (S7)" for "Eastern Equine Encephalitis Disease (D1)" as shown in Fig. 8.

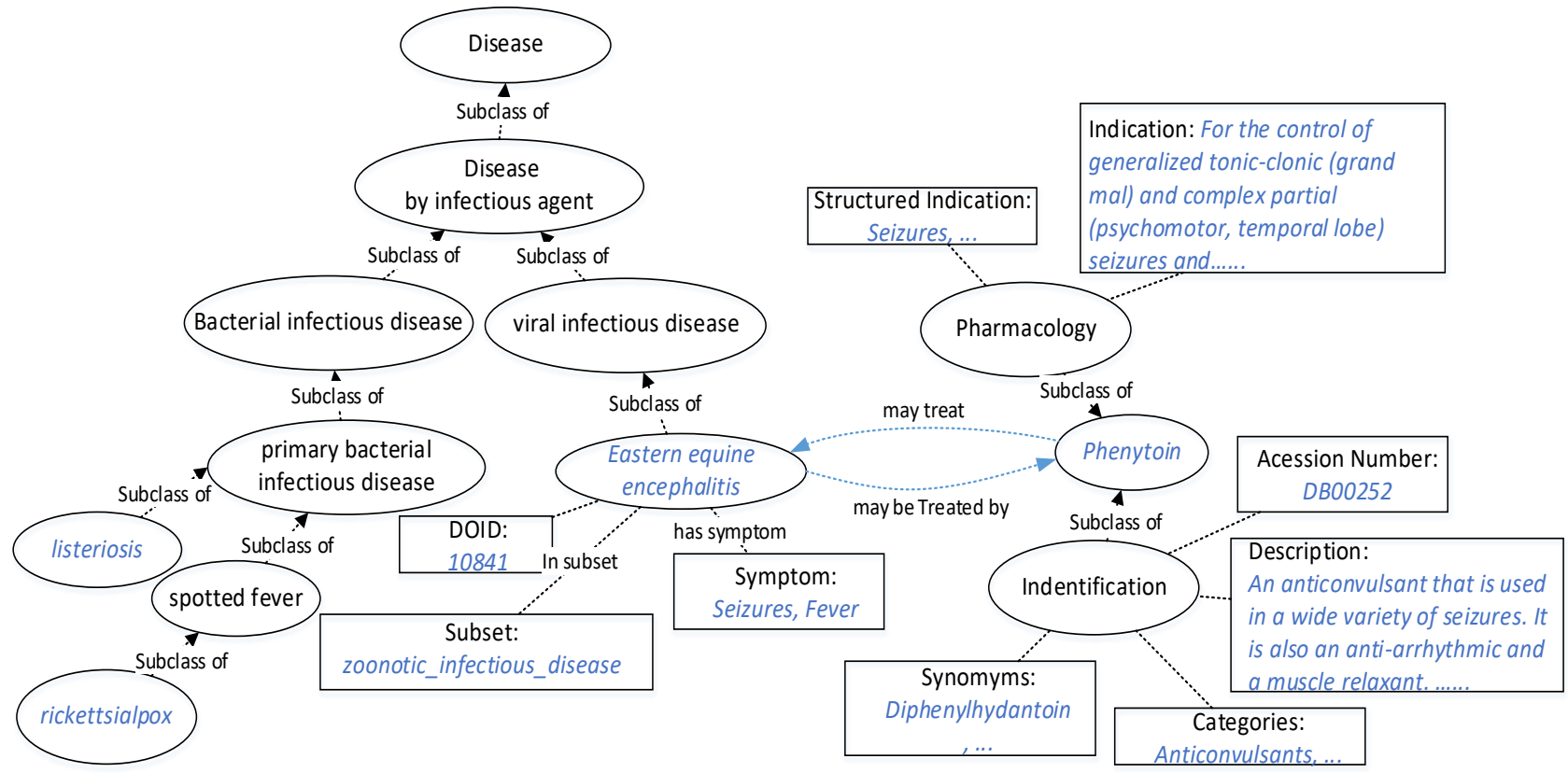

Fig. 8: A merged ontology to treat eastern equine encephalitis 


\section{Evaluation}

After we emerge the proposed merging method, we assured the correctness of the result by evaluating the final merged ontology with the well-known standard collection of Drug, and Disease from Medscape [15]. Medscape offered essential point-of-care drug and disease information for physicians and health-care professionals. With the given example of Eastern Equine Encephalitis treatment, the Medscape collection stated that it can be treated by Phenytoin and Diazepam for Anticonvulsants (Seizure) symptom. The merged ontology remained consistent with the Medscape collection in most cases.

\section{Conclusion and Future Work}

After the result of the heterogeneous ontology merging using our proposed method, we have received a merged ontology that reflected the correct semantic result. However, there are some cases, in our case study, that are inapplicable with the proposed method, e.g. Dengue Fever, and NSAID Drug. In some specific domain, the result shows that it required domain knowledge to determine whether the attribute are relevant or not. However, the evaluation also shows that the merged ontology is correct by comparing with Medscape collection and It was useful for supporting knowledge engineers in merging any domain-specific ontologies together.

For the future work, we found out that the proposed method is able to enhance by participating with other relevant factors. We can also assure the correctness of the proposed method by asking the experts' review on the final merged result. Other than that, the supportive merging tool can be developed in order to facilitate the knowledge engineers in merging digitally and the tool can help in evaluating the applicability in the real work environment.

\section{References}

[1] B. Stumme and G. Gerd, "Creation and Merging of Ontology Top-Levels," Concept. Struct. Knowl. Creat. Commun., pp. 131-145, 2003.

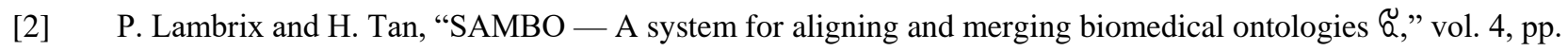
196-206, 2006.

[3] A. Formica, "Ontology-based concept similarity in Formal Concept Analysis," vol. 176, pp. 2624-2641, 2006.

[4] Tom Gruber, "Ontology (Computer Science) - definition in Encyclopedia of Database Systems." [Online]. Available: http://tomgruber.org/writing/ontology-definition-2007.htm. [Accessed: 22-Nov-2016].

[5] "Ontology Development 101: A Guide to Creating Your First Ontology.” [Online]. Available: https://protege.stanford.edu/publications/ontology_development/ontology101-noy-mcguinness.html. [Accessed: 04-May-2018].

[6] "What is an Ontology?" [Online]. Available: http://www.cs.man.ac.uk/ stevensr/onto/node3.html. [Accessed: 04-May-2018].

[7] Y. C. Kiong, S. Palaniappan, and N. A. Yahaya, "Health Ontology System," pp. 1-4, 2011.

[8] S. Amrouch and S. Mostefai, "Survey on the literature of ontology mapping, alignment and merging," 2012 Int. Conf. Inf. Technol. e-Services, ICITeS 2012, 2012.

[9] P. R. Rahm, Erhard; Arnold and Salvatore, "Semantic ontology mappings: How to determine and use them," 2014.

[10] F. Škopljanac-Mačina and B. Blaškovic, "Formal concept analysis - Overview and applications," Procedia Eng., vol. 69, pp. 1258-1267, 2014.

[11] M. Priya and C. A. Kumar, "A survey of state of the art of ontology construction and merging using Formal Concept Analysis," Indian J. Sci. Technol., vol. 8, no. 24, 2015.

[12] G. Stumme and A. Maedche, "FCA-MERGE: Bottom-up merging of ontologies," IJCAI Int. Jt. Conf. Artif. Intell., pp. 225-230, 2001.

[13] L. Guan-yu, L. Shu-peng, and Z. Yan, "Formal concept analysis based ontology merging method," 3rd Int. Conf. Comput. Sci. Inf. Technol., vol. 8, pp. 279-282, 2010.

[14] W. Zhen-Xing and T. Xing-Yan, "Research of Ontology Merging Based on Concept Similarity," Proc. - 2015 7th Int. Conf. Meas. Technol. Mechatronics Autom. ICMTMA 2015, no. 2, pp. 831-834, 2015.

[15] "Latest Medical News, Clinical Trials, Guidelines - Today on Medscape." [Online]. Available: https://www.medscape.com/. [Accessed: 25-Feb-2019]. 